\begin{tabular}{|c|l|}
\hline Title & Stimulus context determines whether non-target stimuli are processed as task-relevant or distractor information \\
\hline Author(s) & Sawaki, Risa; Katay ama, Jun'ichi \\
\hline Citation & $\begin{array}{l}\text { Clinical Neurophysiology, 117(11), 2532-2539 } \\
\text { https://loi.org/10.1016/.Clinph.2006.06.755 }\end{array}$ \\
\hline Issue Date & 2006-11 \\
\hline Doc URL & http://hdl.handle.net/2115/15811 \\
\hline Type & article (author version) \\
\hline File Information & CN117-11.pdf \\
\hline
\end{tabular}

Instructions for use 
Clinical Neurophysiology, 2006, 117, 2532-2539.

\title{
Stimulus context determines whether non-target stimuli are processed as task-relevant or distractor information
}

\author{
Risa SAWAKI ${ }^{\text {a, b }}$ \& Jun'ichi KATAYAMA ${ }^{\text {a }}$ \\ a Graduate School of Education, Hokkaido University, Japan \\ ${ }^{b}$ Research Fellow of the Japan Society for the Promotion of Science, Japan
}

Corresponding author:

Risa SAWAKI

Graduate School of Education, Hokkaido University

Sapporo 060-0811, JAPAN

Tel \& Fax: +81-11-706-3113

e-mail: sawaki@edu.hokudai.ac.jp

Key words:

Event-related potential, Stimulus context, P3a, P3b, Non-target, Three-stimulus oddball paradigm 


\section{Abstract}

Objective: The P300 event-related brain potential (ERP) was elicited using a visual three-stimulus oddball paradigm (standard 0.70, target 0.15 , non-target 0.15 ) to examine how target/standard stimulus context affects non-target processing.

Methods: Target/standard discrimination difficulty (easy or difficult) and non-target /target similarity (similar or dissimilar) were manipulated orthogonally. Participants ( $N$ $=13$ ) were instructed to respond to each infrequent target stimulus by pressing a button.

Results: Target stimuli in all task conditions elicited P3b, which was affected only by the difficulty of target/standard discrimination. When target/standard discrimination was easy, the amplitude of non-target P3 was larger for similar than for dissimilar non-target. In contrast, when target/standard discrimination was difficult, non-target stimuli elicited P3a, the amplitude of which was larger for dissimilar than for similar non-target. Thus, the P300 component for non-target stimuli and the pattern of the effect of target similarity on each P300 component varied as a function of the target/standard stimulus context.

Conclusions: The target/standard stimulus context influences the attentional set for stimulus processing such that it determines whether non-target stimuli are processed as task-relevant or distractor information. 
Significance: The present results are important for understanding the mechanism of cognitive modification in non-target processing. 


\section{Introduction}

Various events occur in the external environment and they may have significant meaning for an organism. Therefore, the appropriate evaluation of events is crucial for successful adaptation to the external environment, and this cognitive process underlies a broad range of human behavior. Furthermore, flexible cognitive modification is also important for survival in a changing environment. Actually, some studies have reported that whether a successful exclusion of irrelevant information from perception occurs (i.e., early selection) or a failure to exclude irrelevant information from perception occurs (i.e., late selection) are dynamically modified by the level of perceptual load of relevant information processing (Lavie, 1995, 2005; Lavie and Tsal, 1994). It is assumed that the context in addition to the physical attributes of events also strongly affects the response to the events. However, the mechanism of cognitive modification in the event evaluation process has not yet been elucidated because this process is complete immediately after the event is detected and interacts with various psychological factors. Event-related brain potential (ERP) is one of the most suitable measurements for investigating such a cognitive mechanism because it has notable temporal resolution and each component has been associated with specific psychological factors (e.g., Otten and Rugg, 2004). 


\section{1. $P 3 b$ and $P 3 a$}

One of the most widely studied ERP components is P300 or P3. This component is usually obtained with the so-called "oddball paradigm”, which is based on the random occurrence of infrequent stimuli that are embedded in a train of frequent standard stimuli. In a classical oddball paradigm, or “two-stimulus” oddball paradigm, a participant has to respond to each infrequent target stimulus by pressing a button or by silent counting. In ERP, target stimuli elicit "P3b", which is a large positive-going potential that has a maximum amplitude over parietal electrode sites with a peak latency of about 300-600 ms, depending on the stimulus modality and task difficulty (Katayama and Polich, 1996a; Picton, 1992). This component has provided a great deal of information about the neural activity that underlies fundamental cognitive operations; e.g., its amplitude reflects the allocation of attentional resources (Humphrey and Kramer, 1994; Kok, 1997,2001; Wickens et al., 1983), and its peak latency reflects the stimulus classification time (Kutas et al., 1977; Magliero et al., 1984; McCarthy and Donchin, 1981).

One variant of the oddball paradigm, the "three-stimulus" oddball paradigm, presents the participant with an additional infrequent non-target stimulus that is inserted into the sequence of target and standard stimuli. When "typical" (i.e., not novel, easily recognized) non-target stimuli are presented, they also elicit a P300 
component that is smaller in amplitude than the target P300, and its latencies and morphologies are similar to those of the target P300 (Katayama \& Polich, 1996b,1999; Pfefferbaum et al., 1980). In contrast, when perceptually "novel” (e.g., colorful, unrecognizable slide) non-target stimuli are presented in a series of more typical (e.g., simple figures) stimuli, they elicit a large positive component that is different from P3b. This component is called "novelty P3" or "P3a”, which has a shorter latency than P3b and has a frontal/central amplitude distribution (Courchesne et al., 1975; Friedman et al., 1993; Squires et al., 1975). This component can be elicited by auditory (Squires et al., 1975), visual (Courchesne et al., 1975), and somatosensory stimuli (Yamaguchi and Knight, 1991). P3a is considered to reflect the capture and orientation of attention towards deviant distractor information, and its amplitude reflects the attentional resources that are allocated for such orientation processing (Berti et al., 2004; Escera et al, 2000; Friedman et al., 2001; Schröger and Wolff, 1998), therefore the amplitude increases as a function of stimulus deviancy (Berti et al., 2004).

\subsection{Stimulus context}

As mentioned above, it has been considered that stimulus novelty is a critical determinant of P3a generation. However, Katayama and Polich (1998) reported that P3a generation could be dynamically modified by the target/standard stimulus context. 
The difficulty of perceptual discrimination between target and standard stimulus was manipulated in an auditory three-stimulus oddball paradigm using "typical" tone stimuli. For the condition in which target/standard discrimination was easy, P300 for a deviant non-target was similar in latency and morphology to that elicited by the target, but showed an appreciably smaller amplitude. In contrast, for the condition in which target/standard discrimination was difficult, P300 for a deviant non-target had a shorter latency and larger amplitude over the frontal/central location compared to target P3b. This component was considered to be "P3a", which has been reported when "novel" non-target stimuli were used (Courchesne et al., 1975; Friedman et al., 1993). Importantly, the degree of non-target deviation from the standard was the same between the easy and difficult conditions. These results showed that the target/standard stimulus context is also a critical factor of P3a generation for non-target. Comerchero and Polich (1999) reported that the target/standard stimulus context determines P3a generation for a typical non-target in the visual modality, and Polich and Comerchero (2003) showed that a deviant typical non-target and novel non-target produced robust and highly similar P300 components when target/standard discrimination was difficult.

\subsection{Present study}

The above previous studies have shown that the target/standard stimulus context 
determines whether or not non-target stimuli elicit P3a. Thus, these findings imply that the target/standard stimulus context affects the processing of non-target. However, the underlying mechanism, in particular the interplay between non-target processing and the target/standard stimulus context, is as yet unclear because previous studies have mainly focused on the issues of stimulus context and P3a generation.

The main purpose of the present study was to clarify how stimulus context affects non-target processing. We hypothesized that the target/standard stimulus context influences the attentional set for stimulus processing such that it determines whether non-target stimuli are processed as task-relevant or distractor information. To examine this hypothesis, the difficulty of target/standard discrimination (easy or difficult) and non-target/target similarity (similar or dissimilar) were manipulated orthogonally. Target/standard discrimination difficulty is a critical determinant of the target/standard stimulus context. Non-target/target similarity could provide information about how non-target stimuli are processed. If non-target stimuli are processed as task-relevant information, they would elicit a target-like P300 and target-similar non-target stimuli would elicit larger amplitude than target-dissimilar non-target stimuli (Azizian et al., 2006; Watson et al., 2005). In contrast, if non-target stimuli are processed as distractor information, they would elicit P3a and target-dissimilar non-target stimuli would elicit larger amplitude than target- similar non-target stimuli (Berti et al., 2004; Courchesne et al., 1975; Friedman et al., 1993). 


\section{Methods}

\subsection{Participants}

Thirteen young adults (6M, 7F) with normal or corrected-to-normal visual acuity and normal color vision served as participants $(M=23.6$ years, $S D=2.4$ years, age range $=21-29$ years). All participants reported being free of neurological or psychiatric disorders and provided written, informed consent.

\subsection{Stimulus and Procedure}

Stimuli were presented in a random series, once every $1 \mathrm{~s}$ with a $100 \mathrm{~ms}$ duration. In each experimental condition, 300 stimuli were presented, consisting of standard, target, and non-target stimuli with probabilities of $.70, .15$, and .15 , respectively. Four task conditions were defined by a combination of two levels of target/standard discrimination difficulty (easy or difficult) and two levels of non-target/target similarity (similar or dissimilar). Target stimuli consisted of small blue circles (approximately $1.15^{\circ} \times 1.15^{\circ}$ ) in all task conditions. Standard stimuli were large blue circles (approximately $2.30^{\circ} \mathrm{x} 2.30^{\circ}$ ) in the easy tasks and small blue circles (approximately $1.30^{\circ} \times 1.30^{\circ}$ ) that were slightly larger than the target circles in the 
difficult tasks. Non-target stimuli were small blue squares (approximately $1.00^{\circ} \mathrm{x}$ $1.00^{\circ}$ ) for similar non-target conditions and large red squares (approximately $2.00^{\circ} \mathrm{x}$ $2.00^{\circ}$ ) for dissimilar non-target conditions. The stimuli and their sizes in each task condition are summarized in Figure 1. The participants were asked to respond to the target stimuli by pushing a button with the right thumb as quickly as possible. Each experimental condition was divided into two experimental blocks and each block lasted $2.5 \mathrm{~min}$. The order of the four conditions was randomized across participants.

Insert Figure 1 around here.

\subsection{Recording Condition}

Electroencephalographic (EEG) activity was recorded with an electrode cap using Ag/AgCl electrodes from 30 electrode sites at Fp1, Fp2, Fz, F3, F4, F7, F8, FCz, FC3, FC4, FT7, FT8, Cz, C3, C4, T7, T8, CPz, CP3, CP4, TP7, TP8, Pz, P3, P4, P7, P8, Oz, O1, and O2 according to the modified 10-20 System. Voltage changes caused by eye movements and blinks were monitored with EEG recordings from forehead sites (Fp1, Fp2) and electrooculograms (EOG) recording from bipolar electrodes placed at the left 
and right canthi. A common reference electrode was placed at the nose tip, and impedance was kept at $10 \mathrm{k} \Omega$ or less. The EEG and EOG signals were amplified with a bandpass of $0.01-30 \mathrm{~Hz}$ and digitized at $250 \mathrm{~Hz}$. Averaging epochs were $900 \mathrm{~ms}$, beginning 100 ms before stimulus onset. Waveforms were averaged off-line, such that trials with a response error or those in which the EEG or EOG exceeded $\pm 100 \mu \mathrm{V}$ were rejected automatically.

\subsection{Data Analyses}

The P300 component was defined as the largest positive-going peak that occurred within the time window between 300 and 700 ms after stimulus presentation. Since the focus of the present study was the effect of the stimulus context on target and non-target stimuli, only P300 components in response to these stimuli were assessed. To estimate the reliable peak amplitude and peak latency, averaged waveforms were digitally low-pass filtered at $8 \mathrm{~Hz}$ (24 dB/octave) with a finite impulse response (FIR), zero-phase filter. Peak amplitude was measured relative to the prestimulus baseline, and peak latency was measured from the time of stimulus onset. To reduce the number of statistical comparisons, the data from only 5 midline electrodes (Fz, FCz, Cz, CPz, $\mathrm{Pz}$ ) were analyzed statistically. All analyses of variance (ANOVAs) employed Greenhouse-Geisser corrections to the degrees of freedom, and only the corrected 
probability values are reported.

\section{Results}

\subsection{Behavioral Performance}

The behavioral data are summarized in Table 1. A two-factor (2 Target/Standard Discrimination Difficulties x 2 Non-target/Target Similarities) analysis of the reaction time to the target found that the reaction time in easy tasks was shorter than that in difficult tasks, $F(1,12)=85.9, p<.001$. The same analysis found that the hit rate was higher in easy tasks than in difficult tasks, $F(1,12)=33.2, p<.001$. Furthermore, the false positive rate for the standard in difficult tasks was higher than that in easy tasks, $F(1,12)=9.45, p<.01$. These results indicate that RT, hit rate, and the false positive rate for the standard differed as a function of the difficulty of target/standard discrimination. An analysis of the false positive rate for the non-target revealed a main effect of Non-target/Target Similarity, $F(1,12)=6.137, p<.03$, and a significant interaction, $F(1,12)=8.1, p<.02$. This interaction indicates that non-target/target similarity had no effect in the difficult task, whereas the false positive rate for the similar non-target was higher than that for the dissimilar non-target in the easy task ( $p$ $<.02)$. 
Insert Table 1 around here.

\subsection{ERP Analyses}

Figure 2 shows the grand averaged ERPs from the five midline electrodes for the standard, target, and non-target stimuli in each condition. Figure 3 shows topographic maps taken at the latency of the peak P300 amplitude for the target and non-target stimuli in each task condition.

Insert Figures 2 and 3 around here.

\subsubsection{P300 Amplitude}

The data from the midline electrodes (Figure 4) were assessed with a four-factor (2 Stimulus Types x 2 Target/Standard Discrimination Difficulties x 2 Non-target/Target Similarities x 5 Electrodes) ANOVA. Table 2 summarizes the results of this analysis. 
Main effects of Stimulus Type [S], Non-target/Target Similarity [N], and Electrode [E] were obtained, but were further modified by interaction effects, as is obvious from Figure 4. P300 amplitudes at central/parietal electrode sites were larger for the target than the non-target stimuli yielding a significant two-way interaction between $\mathrm{S} x \mathrm{E}$. Other interactions arose from the manipulation of target/standard discrimination difficulty [T]. Significant $S \times T, T \times N$, and $S \times T \times N$ interactions indicate that target/standard discrimination difficulty and non-target/target similarity provided different effects for the target and non-target. The amplitude of non-target P300 was larger in the similar condition than in the dissimilar condition for the easy task ( $p$ $<.06$ ), but was larger in the dissimilar condition than in the similar condition for the difficult task $(p<.001)$, whereas the target P300 amplitude was smaller in the difficult task than in the easy task $(p<.001)$, regardless of the non-target/target similarity. Further, significant $\mathrm{T} \times \mathrm{E}$ and $\mathrm{S} \times \mathrm{T} \times \mathrm{E}$ interactions were observed. The separate analyses showed the $\mathrm{T} \times \mathrm{E}$ interaction was significant for non-target, whereas the interaction was not significant for target. These results indicate that the manipulation of target/standard discrimination difficulty provided a modulation of non-target P300 scalp distribution, resulting in clear differences between non-target P300s in the easy and difficult tasks. P300 amplitude for non-target was smaller than that for target at all electrode sites when target/standard discrimination was easy, whereas P300 amplitude for non-target was larger than that for target at Fz, FCz, and $\mathrm{Cz}$ electrode sites when 
target/standard discrimination was difficult $(p<.001, p<001$, and $p<.02)$, indicating that the non-target P300 shifted to a frontal-central distribution in the difficult tasks.

Insert Table 2 and Figures 4 and 5around here.

\subsubsection{P300 Latency}

Mean P300 latencies from the target $(\mathrm{Pz})$ and non-target $(\mathrm{Cz})$ stimuli in each task condition are illustrated in Figure 5. A three-factor (2 Stimulus Types $x 2$ Target/Standard Discrimination Difficulties $x 2$ Non-Target/Target Similarities) analysis of the latency found significant main effects of Stimulus Type, $F(1,12)=$ 25.2, $p<.001$, Target/Standard Discrimination Difficulty, $F(1,12)=40.5, p<.001$, and Non-target/Target Similarity, $F(1,12)=7.1, p<.03$. There was also significant two-way interaction between Stimulus Type and Target/Standard Discrimination Difficulty, $F(1,12)=21.6, p<.001$. Post-hoc comparisons revealed that there was no difference between target and non-target P300 latencies when target discrimination was easy, whereas the target P300 latency was longer than the non-target P300 latency when target discrimination was difficult $(p<.001)$. 


\section{Discussion}

When it was difficult to discriminate the target from the standard stimuli, the reaction time and false positive rate for the standard increased and the hit rate decreased compared to when target discrimination was easy. Thus, the results confirmed that the difficulty of discrimination was successfully manipulated by varying the physical size of the target and standard stimuli. Although the overall false positive rate for non-targets was low, the interaction between target/standard discrimination difficulty and non-target/target similarity was significant. When target/standard discrimination was difficult, the similarity between non-target and target had no effect on the false positive rate for non-target. In contrast, when that discrimination was easy, the false positive rate for similar non-target was increased compared to the dissimilar non-target, which suggests that non-target/target similarity had a significant effect in easy tasks and an inappropriate response to non-target tended to occur when the non-target was similar to the target. This effect will be discussed in detail below.

\subsection{Target $P 300$}

The target stimuli in all task conditions elicited a P300 component that was largest 
over the central/parietal and midline electrode sites. This component is assumed to be “P3b”. The target P3b was significantly affected by the difficulty of target/standard discrimination, such that when target/standard discrimination was difficult, amplitude decreased and latency increased. This outcome is highly consistent with many reports using a classic oddball paradigm (e.g., Polich, 1986; Verleger, 1997), and a three-stimulus oddball paradigm (Katayama and Polich, 1998). It is assumed that the difficulty of discrimination increased an equivocation which could result from weaker memory representations or lower internal discriminability of target events (Kok, 2001). Another possibility is the increased latency jitter which arises from the task difficulty. The decreased amplitude in difficult tasks would be due to these factors. The results confirmed that the difficulty of discrimination was successfully manipulated in the present study. In contrast, the target P300 was not affected by non-target/target similarity, and this outcome agrees with the present behavioral results. Katayama \& Polich (1998) also reported that the magnitude of the deviation of non-target from standard had no effect on target P300. Taken together, these results suggest that target processing is independent of the non-target properties such as similarity to the target or degree of deviation.

\subsection{Non-target $P 300$}


Non-target stimuli elicited P300 components in all conditions, although their scalp distribution, amplitudes and latencies varied with the difficulty of target/standard discrimination and the similarity to the target.

In easy tasks, both similar and dissimilar non-target stimuli elicited P300s that were smaller in amplitude than the target P300, and their latencies were similar to those of the target P300. These outcomes are highly consistent with previous reports using a three-stimulus oddball paradigm (Katayama \& Polich, 1996a, 1996b, 1999; Pfefferbaum et al., 1980), and one of which demonstrated that the stimulus probability also affects non-target P300 amplitude in a manner similar to target P300 amplitude (Katayama \& Polich, 1996b). This type of non-target P300s have been labeled "P3b" (Katayama \& Polich, 1996b) or "no-go P300" (Polich, 2003), and there is no consensus in the literature on the appropriate nomenclature for this component. For the sake of simplicity, "non-target P3" will be used in the present article. In the comparison of non-target P3s between similar and dissimilar non-target, the amplitude for similar non-target was larger than that for dissimilar non-target, i.e., the amplitude was larger as a function of task relevance. In addition, the behavioral data revealed the highest commission error for the non-target was observed in the easy/similar condition. Therefore, these similarity effects for non-target P3 and behavioral data suggest that non-target was processed as task-relevant information when target/standard discrimination was easy. 
In difficult tasks, ERP data showed that P300 components for non-target had shorter latency and larger amplitude, especially at anterior electrode sites, compared with the target P300 component. This outcome closely agreed with previous studies which reported that highly deviant non-target elicited a P3a component when target/standard discrimination was difficult (Comerchero and Polich, 1998, 1999; Katayama and Polich, 1998; Polich and Comerchero, 2003). Therefore, these non-target P300s are considered to be "РЗa". P3a reflects the capture and orientation of attention toward deviant distractor information. Importantly, not only dissimilar but also similar non-target elicited a P3a component, and the amplitude for the dissimilar non-target was larger than that for the similar non-target, which indicates that a more deviant non-target was allocated more attentional resources. Therefore, this pattern of the similarity effect suggests that non-target was processed as distractor information when target/standard discrimination was difficult.

\subsection{Stimulus context and non-target processing}

Previous studies have reported that P3a generation could be dynamically modified by the target/standard stimulus context (Comerchero and Polich, 1999; Katayama and Polich, 1998; Polich, 2003). This finding indicated that the target/standard stimulus context influences the processing of non-target. However, it is unclear how the 
stimulus context affects non-target processing. The main purpose of the present study was to clarify this issue.

When target/standard discrimination was easy, a target-similar non-target provided larger amplitude of non-target P3 compared with a dissimilar non-target. This effect of non-target/target similarity on the non-target P3 amplitude confirmed that non-target was estimated in the same manner as target, at least when target/standard discrimination was easy. In easy tasks, an infrequent target stimulus could be easily discriminated from a frequent standard stimulus sequence. Therefore, under this stimulus context, it is considered that the detection of deviation in the visual stimulus sequence is enough to detect the target stimulus and it would be of benefit to keeping attentional resource. Many literatures have demonstrated that attentional set could bias the cognitive process in such a way that one could reliably detect or identify the target events (e.g., Bacon and Egeth, 1994; Folk et al, 1992). The stimulus context would thus modulate an attentional set toward the change from the standard, and then the presentation of infrequent stimuli would trigger task-relevant processing. Accordingly, non-target stimuli would be processed as task-relevant information because not only target but also non-target stimuli appear infrequently in the visual stimulus sequence.

In contrast, when target/standard discrimination was difficult, non-target stimuli elicited P3a. Moreover, a target-dissimilar non-target provided a larger P3a amplitude compared with a similar non-target. This effect of non-target/target similarity on the 
P3a amplitude confirmed that non-target was processed as distractor information when target/standard discrimination was difficult. In difficult tasks, frequent standard stimuli are perceptually similar to the target stimuli. Therefore, it is considered that the detection of deviation in the visual stimulus sequence is not appropriate to detect the target and the stimulus context would thus modulate the attentional set toward cautious discrimination between slightly larger circle (standard) and slightly smaller circle (target). The non-target stimuli in the present study had a different shape (square) than target and standard stimuli (circle). Therefore, non-target stimuli would not be processed as task-relevant but processed as distractor information because they disturb the attentional set toward the cautious discrimination between standard and target stimuli. Hence, they might be dismissed immediately from the evaluation of task-relevance.

In summary, the P300 ERP for non-target was altered as a function of the difficulty of target/standard discrimination. Non-target stimuli are processed as task-relevant information under the easy target/standard stimulus discrimination context. In contrast, they are processed as distractor information under the difficult target/standard stimulus discrimination context. The results of the present study suggest that the target/standard stimulus context influences the attentional set, which determines non-target processing. 


\section{References}

Azizian A, Freitas AL, Watson TD, Squires NK. Electrophysiological correlates of categorization: P300 amplitude as index of target similarity. Biol Psychol 2006;71:278-288.

Bacon WF, Egeth HE. Overriding stimulus-driven attentional capture. Percept Psychophys 1994;55:485-496.

Berti S, Roeber U, Schröger E. Bottom-up influences on working memory: Behavioral and electrophysiological distraction varies with distractor strength. Exp Psychol 2004;51:249-257.

Comerchero MD, Polich J. P3a, perceptual distinctiveness, and stimulus modality. Cogn Brain Res 1998;7:41-48.

Comerchero MD, Polich J. P3a and P3b from typical auditory and visual stimuli. Clin Neurophysiol 1999;110:24-30.

Courchesne E, Hillyard SA, Galambos R. Stimulus novelty, task relevance and the visual evoked potential in man. Electroencephalogr Clin Neurophysiol 1975;39:131-143.

Escera C, Alho K, Schröger E, Winkler I. Involuntary attention and distractibility as evaluated with event-related brain potentials. Audiol Neurootol 2000;5:151-166.

Folk CL, Remington RW, Johnston JC. Involuntary covert orienting is contingent on 
attentional control settings. J Exp Psychol Hum Percept Perform 1992;18:1030-1044.

Friedman D, Simpson G, Hamberger M. Age-related changes in scalp topography to novel and target stimuli. Psychophysiology 1993;30:383-396.

Friedman D, Cycowicz YM, Gaeta H. The novelty P3: an event-related brain potential (ERP) sign of the brain's evaluation of novelty. Neurosci Biobehav Rev 2001;25:355-373.

Humphrey DG, Kramer AF. Toward a psychophysiological assessment of dynamic changes in mental workload. Hum Factors 1994;36:3-26.

Katayama J, Polich J. P300 from one-, two-, and three-stimulus auditory paradigms. Int J Psychophysiol 1996a;23:33-40.

Katayama J, Polich J. P300, probability, and the three-tone paradigm. Electroencephalogr Clin Neurophysiol 1996b;100:555-562.

Katayama J, Polich J. Stimulus context determines P3a and P3b. Psychophysiology 1998;35:23-33.

Katayama J, Polich J. Auditory and visual P300 topography from a 3 stimulus paradigm. Clin Neurophysiol 1999;110:463-468.

Kok A. Event-related-potential (ERP) reflections of mental resources: A review and synthesis. Biol Psychol 1997;45:19-56.

Kok A. On the utility of P3 amplitude as a measure of processing capacity. 
Psychophysiology 2001;38:557-577.

Kutas M, McCarthy G, Donchin E. Augmenting mental chronometry: The P300 as a measure of stimulus evaluation time. Science 1977;197:792-795.

Lavie N. Perceptual load as a necessary condition for selective attention. J Exp Psychol Hum Percept Perform 1995;21:451-468.

Lavie N. Distracted and confused? : Selective attention under load. Trends Cogn Sci 2005;9:75-82.

Lavie N, Tsal Y. Perceptual load as a major determinant of the locus of selection in visual attention. Percept Psychophys 1994;56:183-197.

Magliero A, Bashore TR, Coles MGH, Donchin E. On the dependence of P300 latency on stimulus evaluation processes. Psychophysiology 1984;21:171-186.

McCarthy G, Donchin E. A metric for thought: A comparison of P300 latency and reaction time. Science 1981;211:77-80.

Otten LJ, Rugg MD. Interpreting event-related brain potentials. In: Handy TC, editor. Event-related Potentials: A Methods Handbook. Cambridge, MA: MIT Press, 2004:3-16.

Pfefferbaum A, Ford JM, Roth WT, Kopell BS. Age-related changes in auditory event-related potentials. Electroencephalogr Clin Neurophysiol 1980;49:266-276.

Picton TW. The P300 wave of the human event-related potential. J Clin Neurophysiol 1992;9:456-479. 
Polich J. Attention, probability, and task demands as determinants of P300 latency from auditory stimuli. Electroencephalogr Clin Neurophysiol 1986;63:251-259.

Polich J. Overview of P3a and P3b. In: Polich J, editor. Detection of Change: Event-Related Potential and fMRI Findings. Boston: Kluwer Academic Press, 2003:83-98.

Polich J, Comerchero MD. P3a from visual stimuli: Typicality, task, and topography. Brain Topogr 2003;15:141-152.

Schröger E, Wolff C. Behavioral and electrophysiological effects of task-irrelevant sound change: A new distraction paradigm. Cogn Brain Res 1998;7:71-87.

Squires NK, Squires KC, Hillyard SA. Two varieties of long-latency positive waves evoked by unpredictable auditory stimuli in man. Electroencephalogr Clin Neurophysiol 1975;38:387-401.

Verleger R. On the utility of P3 latency as an index of mental chronometry. Psychophysiology 1997;34:131-156.

Watson TD, Azizian A, Berry S, Squires NK. Event-related potentials as an index of similarity between words and pictures. Psychophysiology 2005;42:361-368.

Wickens C, Kramer A, Vanasse L, Donchin E. Performance of concurrent tasks: A psychophysiological analysis of the reciprocity of information processing resources. Science 1983;221:1080-1082.

Yamaguchi S, Knight RT. P300 generation by novel somatosensory stimuli. 
Electroencephalogr Clin Neurophysiol 1991;78:50-55. 
Table 1. Mean Reaction Times and Performance Rates for Each Task Condition

Target/standard discrimination Easy Difficult

Non-target/target similarity Similar Dissimilar Similar Dissimilar

$\begin{array}{lcccc}\text { Reaction time (ms) } & 387 & 365 & 472 & 471 \\ S D & 35 & 38 & 43 & 40 \\ & & & & \\ & & & & \\ \text { Hit targets (\%) } & 99.2 & 99.7 & 86.2 & 84.4 \\ S D & 1.7 & 1.2 & 10.8 & 9.9\end{array}$

False positive (\%)

$\begin{array}{lcccc}\text { Standard } & 0.04 & 0.04 & 1.02 & 1.91 \\ \text { SD } & 0.14 & 0.14 & 1.69 & 2.74 \\ & & & & \\ & & & & \\ \text { Non-target } & 1.77 & 0.00 & 0.17 & 0.17 \\ \text { SD } & 2.24 & 0.00 & 0.61 & 0.61\end{array}$


Table 2. Summary of the Four-Factor Analysis of Variance (2 Stimulus Types $\mathrm{x}$ Target/Standard Discrimination Difficulties $\times 2$ Non-target/Target Similarity $\times 5$ Electrodes) Performed on the P300 Peak Amplitude

\begin{tabular}{|c|c|c|c|}
\hline Source $(d f)$ & $F$ & $p$ & $\varepsilon$ \\
\hline Stimulus type $(1,12)$ & 10.8 & .007 & - \\
\hline Target/standard discrimination difficulty $(1,12)$ & - & - & - \\
\hline Non-target/target similarity $(1,12)$ & 6.6 & .02 & - \\
\hline Electrode $(4,48)$ & 21.6 & .001 & .36 \\
\hline $\mathrm{S} \times \mathrm{T}(1,12)$ & 74.9 & .001 & - \\
\hline $\mathrm{S} \times \mathrm{N}(1,12)$ & - & - & - \\
\hline $\mathrm{S} x \mathrm{E}(4,48)$ & 69.0 & .001 & .38 \\
\hline $\mathrm{T} \times \mathrm{N}(1,12)$ & 28.1 & .001 & - \\
\hline $\mathrm{T} \times \mathrm{E}(4,48)$ & 5.4 & .01 & .52 \\
\hline $\mathrm{N} x \mathrm{E}(4,48)$ & - & - & - \\
\hline $\mathrm{S} \times \mathrm{T} \times \mathrm{N}(1,12)$ & 31.7 & .001 & - \\
\hline $\mathrm{S} \times \mathrm{T} \times \mathrm{E}(4,48)$ & 4.7 & .02 & .53 \\
\hline$S \times N \times E(4,48)$ & - & - & - \\
\hline $\mathrm{T} \times \mathrm{N} \times \mathrm{E}(4,48)$ & - & - & - \\
\hline $\mathrm{S} \times \mathrm{T} \times \mathrm{N}$ x E $(4,48)$ & - & - & - \\
\hline
\end{tabular}




\section{Figure Legends}

Figure 1. Stimulus characteristics (probability, shape, and size) for each task condition. Color was blue except for red dissimilar non-target.

Figure 2. Grand averaged ERPs for each condition from five midline electrode sites $(N=13)$.

Figure 3. Topographic maps taken at P300 peak latency for the target and non-target stimuli in each task condition.

Figure 4. Mean P300 peak amplitude from the target and non-target in each task condition as a function of midline electrode site.

Figure 5. Mean P300 peak latencies from the target $(\mathrm{Pz})$ and non-target $(\mathrm{Cz})$ stimuli in each task condition. 


\begin{tabular}{|c|c|c|c|c|}
\hline \multirow{2}{*}{$\begin{array}{c}\text { Target/standard } \\
\text { discrimination } \\
\text { Non-target/target } \\
\text { similarity }\end{array}$} & \multicolumn{2}{|c|}{ Easy } & \multicolumn{2}{|c|}{ Difficult } \\
\hline & Similar & Dissimilar & Similar & Dissimilar \\
\hline \multicolumn{5}{|l|}{ Standard $(p=.70)$} \\
\hline \multicolumn{5}{|l|}{ Target $(p=.15)$} \\
\hline & $3.14 \mathrm{~cm}^{2}$ & $3.14 \mathrm{~cm}^{2}$ & $3.14 \mathrm{~cm}^{2}$ & $3.14 \mathrm{~cm}^{2}$ \\
\hline \multicolumn{5}{|l|}{ Non-target $(p=.15)$} \\
\hline & $3.14 \mathrm{~cm}^{2}$ & $12.56 \mathrm{~cm}^{2}$ & $3.14 \mathrm{~cm}^{2}$ & $12.56 \mathrm{~cm}^{2}$ \\
\hline
\end{tabular}

Figure 1 


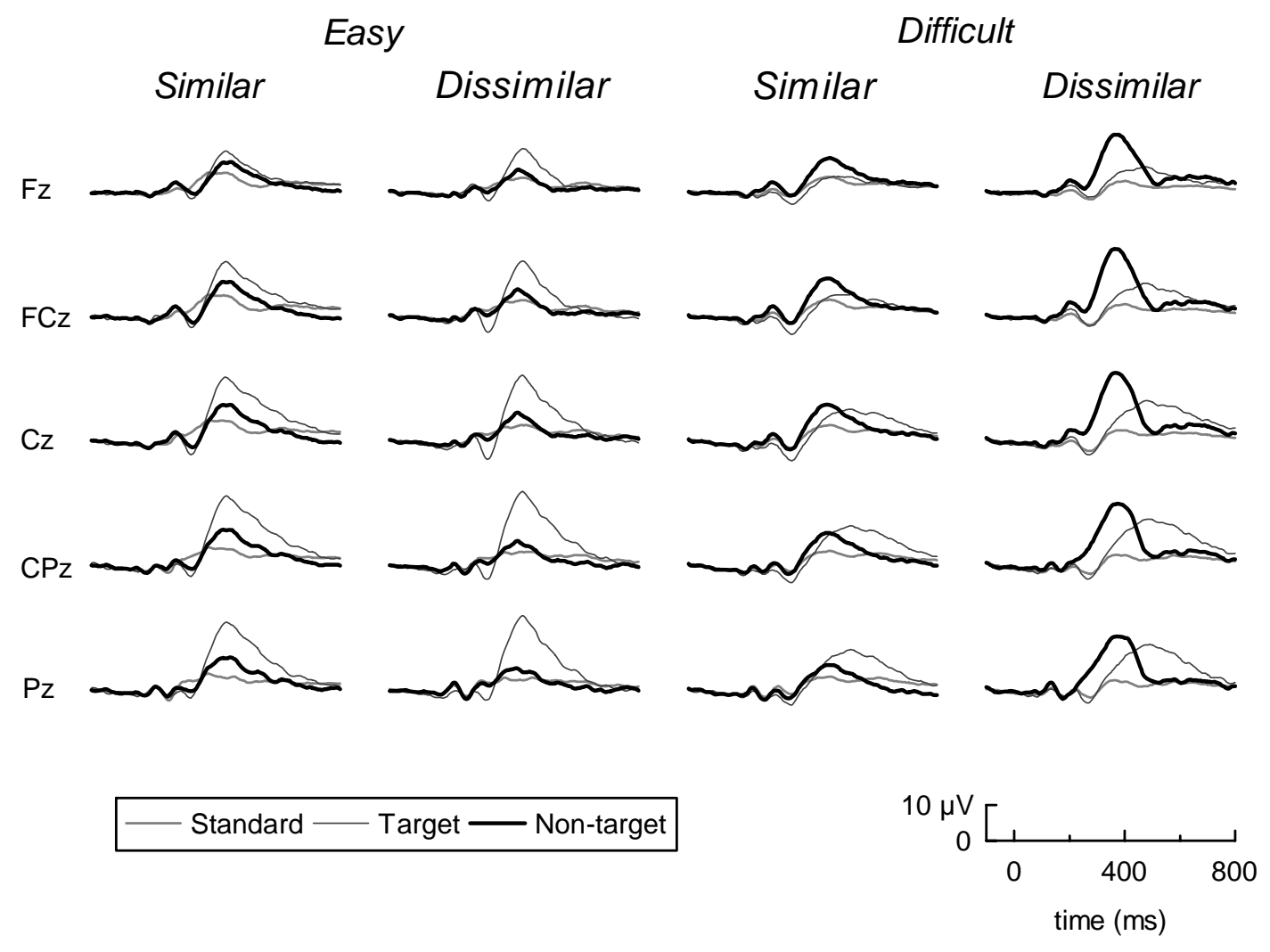

Figure 2 


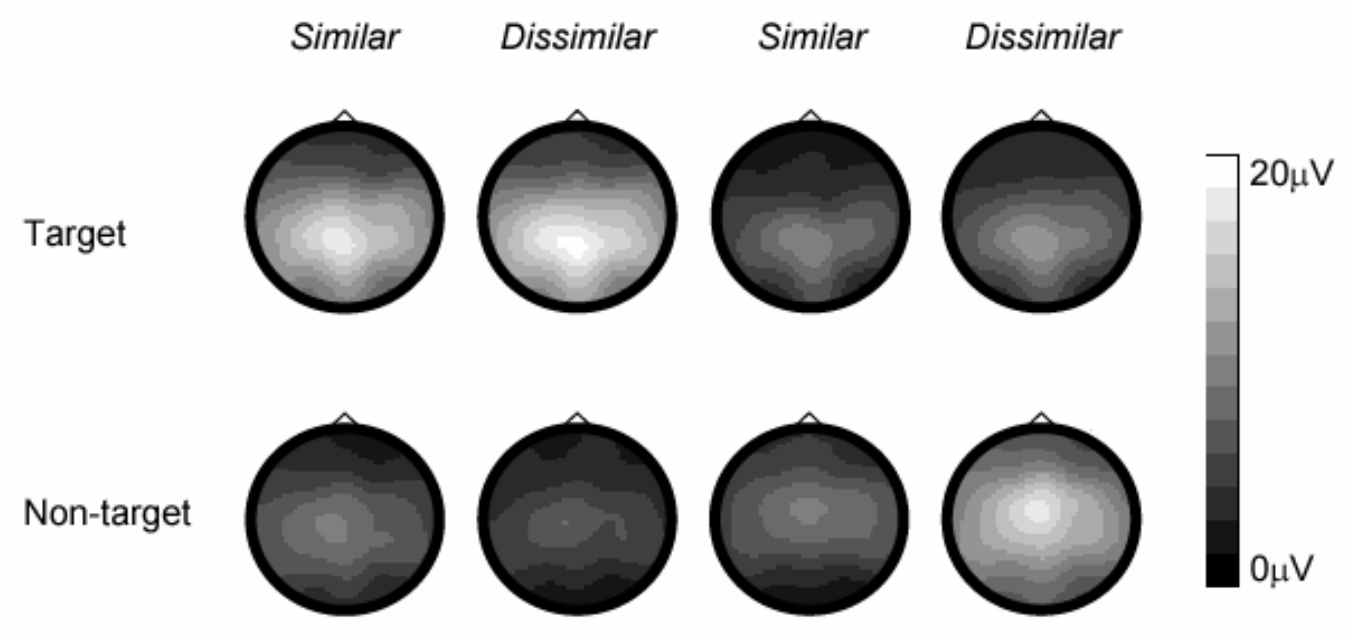

Figure 3 
Similar

Dissimilar
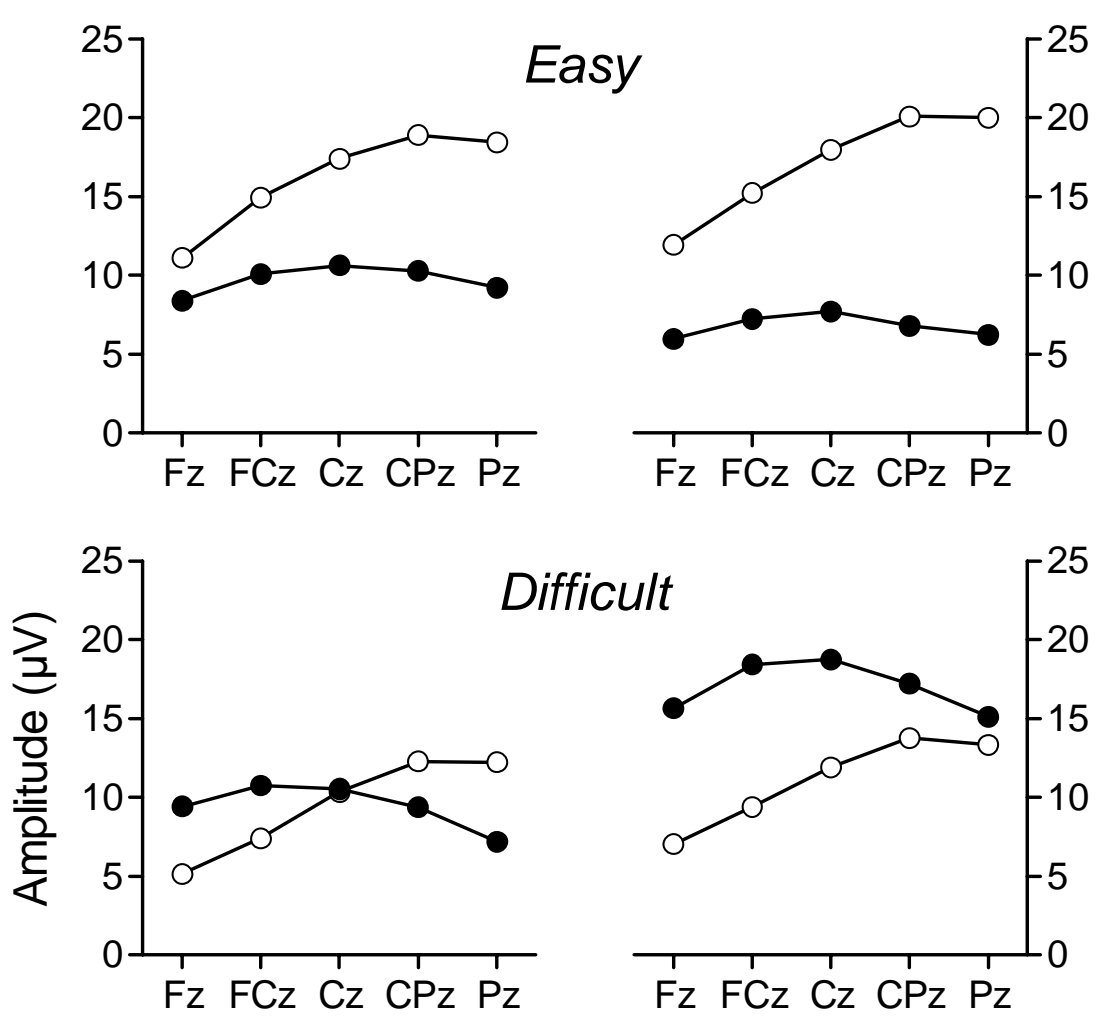

Electrode

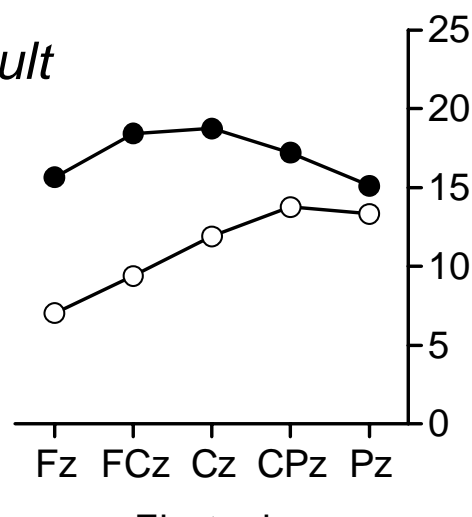

Electrode

$-\circ-$ Target $\longrightarrow$ Non-target

Figure 4 
Easy

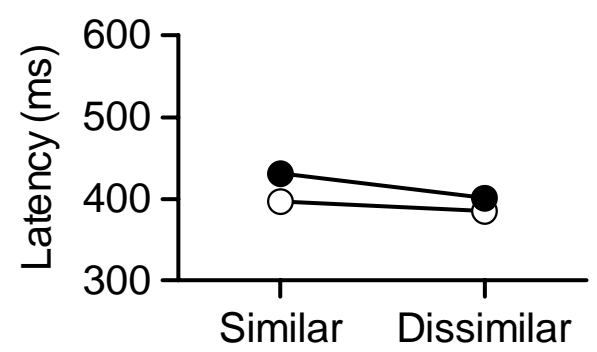

Difficult

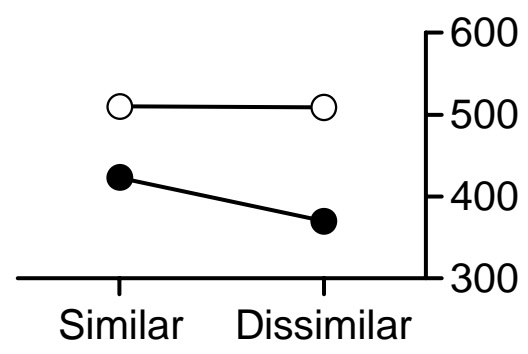

- - Target $\multimap-$ Non-target

Figure 5 